\title{
Finite element analysis of no-tension structures as a topology optimization problem
}

\author{
Matteo Bruggi
}

Received: date / Accepted: date

\begin{abstract}
An alternative numerical approach is presented for the analysis of no-tension masonry-like solids. Whereas most of the strategies available in the literature resort to non-linear finite element techniques, the proposed approach re-formulates the problem within the framework of topology optimization. The equilibrium of a two-dimensional no-tension body is found searching for the distribution of an equivalent orthotropic material, in which tensile principal stresses are not allowed by prescribing negligible stiffness in the relevant direction, such that the potential energy of the solid is minimized. Unlike many conventional approaches that deal with the tough non-linearity of the problem through step-wise incremental analyses, the proposed method efficiently solves the effect of compatible loads through a one-shot energy-based optimization. Analytical and numerical benchmarks from the literature are investigated to assess the effectiveness of the proposed procedure and to discuss convergence features and possible applications inspired by the limit analysis of masonry-like structures.
\end{abstract}

Keywords topology optimization · orthotropic materials $\cdot$ no-tension materials $\cdot$ masonry-like solids

\section{Introduction}

Among the methods for the analysis of masonry-like solids, the no-tension model allows for a preliminary description of the mechanical behavior of a structure

Matteo Bruggi

Department of Civil and Environmental Engineering

Politecnico di Milano, I20133, Milano, Italy

Tel.: + 39-02-23994232

Fax: +39-02-23994220

E-mail: matteo.bruggi@polimi.it based on the assumption that the stress tensor is negative semidefinite and depends linearly upon the elastic part of the strain. A positive semidefinite inelastic strain accounts for scattered cracking, see e.g. Del Piero (1989). This approach follows the well-known rationale that considers masonry as a material with a negligible strength in tension, see e.g. the successful application of the methods of limit analysis to arches and vaults in Heyman (1966).

Notwithstanding the apparent simplicity of the notension model, its numerical treatment is not trivial even dealing with the analysis of elementary cases. A solution exists depending on the compatibility of the external loads with respect to the no-tension assumption and often involves discontinuous stress and displacement fields. Additionally, the displacement field may be indeterminate even in cases where a unique equilibrated solution is found, see e.g. Lucchesi et al. (2000). As reviewed in Alfano et al. (2000), the rate of convergence of the analysis of no-tension materials is, in general, rather poor and the tolerance adopted to stop the iterative process is much lower than that commonly employed in plasticity computations. The work by Cuomo and Ventura (2000) reports an exhaustive overview of the robust numerical approaches that have been proposed in the literature to cope with the inherent nonlinearity of the considered problem and to overcome the particular issues related to the no-tension constraint, see also Baratta and Corbi (2010b). Total displacement formulations and displacement rate approaches (Romano and Sacco 1984; Genna 1994; Lucchesi et al. 1995; Alfano et al. 2000) have been implemented during the last decades, as well as complementary formulations written in terms of stresses (Maier and Nappi 1990; Cuomo and Ventura 2000). In general, these approaches require step-wise analyses to cope with the 
(non-smooth) rate constitutive equations peculiar to the no-tension assumption, which are suitably regularized by means of ad hoc numerical strategies.

Alternatively, one may deal with the analysis of a no-tension solid resorting to the minimization of the elastic strain energy, whose derivation under the plane stress assumption was originally provided in Del Piero (1989). Following this approach, Angelillo et al. (2010) implements a numerical strategy that solves the equilibrium of two-dimensional no-tension bodies through the minimization of the potential energy with respect to the unknown displacement field. A descent method is adopted to minimize the arising unconstrained energy functional, thus overcoming the numerical difficulties related to the enforcement of the no-tension behavior.

The approach proposed in the present contribution moves from the mainstream of energy-based methods to re-formulate the analysis of no-tension solids as a topology optimization problem.

Some conventional formulations of topology optimization distribute a prescribed amount of isotropic material in such a way that the strain energy is minimized. This has the aim of providing the stiffest design among all possible layouts, see e.g. Bendsøe and Kikuchi (1988). The distribution of material is governed by the adoption of suitable interpolations that are ideally conceived to penalize the elastic constants depending on the minimization unknown, as in the element-wise constant approximation implemented e.g. by Bendsøe and Sigmund (1999) or in the pointwise density-based interpolation adopted by Kang and Wang (2011) and Luo et al. (2013). In the case of bodies made of anisotropic materials the design variables include the material orientation, see e.g. Eschenauer and Olhoff (2001) and Foldager et al. (1998). Three different approaches were originally developed to cope with this problem: the strainbased method (Pedersen 1989, 1990; Cheng and Pedersen 1997), the stress-based method (Suzuki and Kikuchi 1991; Diaz and Bendsøe 1992; Cheng and Kikuchi 1994) and the energy-based method (Luo and Gea 1998). These approaches take into account the effect of the change in strain and stress due to the variation in the material orientation, thus providing effective formulations for the optimal design of structures made of orthotropic materials. A general optimality condition that arises when the structural compliance has to be minimized under given static and kinematic boundary conditions, is that the stress and strain tensors have to locally share the same principal directions. This result was first obtained by Pedersen (1989), for twodimensional orthotropic solids, and later extended by Rovati and Taliercio (2003) to three-dimensional orthotropic bodies.
Alternative approaches have been developed in the literature of topology optimization to take into account enforcements on the effective material strength, mainly based on extensions of the SIMP law to cope with stress constraints, see in particular Rozvany et al. (1992), Duysinx and Bendsøe (1998), Le et al. (2010). Stressbased design has also been investigated dealing with structural composites, as in the case of the pioneering contribution by Swan and Arora (1997). Drawbacks of the pure compliance-based optimization have been recently documented in the case of isotropic materials exhibiting a non-symmetric behavior in tension and compression, see e.g. Luo and Kang (2012), Bruggi and Duysinx (2012), Querin et al. (2010). Ad hoc procedures can be implemented to cope with the optimal design of truss-like structures made of isotropic material even for an extreme unequal behavior in tension and compression, see e.g. Cai (2011), Ananiev (2005) and Bruggi and Duysinx (2013).

Within the above framework, this contribution aims at solving the equilibrium of a two-dimensional notension body searching for the distribution of an equivalent orthotropic material, exhibiting negligible stiffness for any direction along which a tensile principal stress must be prevented, such that the potential energy of the solid is minimized. Indeed, as shown e.g. in Bendsøe and Sigmund (2003), a compliance-based optimization may be employed as an energy-based analysis tool by simply removing the constraint on the available amount of material. Two sets of density unknowns are defined in order to control the stiffness of the equivalent composite along its symmetry axes, which should be oriented along the principal stress directions of the no-tension body. A suitable material interpolation law is proposed to recover the conventional isotropic model under biaxial compression, while discarding the stiffness contributions that are relevant to any principal tensile stress direction. An ad hoc penalization of the energy contributions involving tensile stresses is adopted to build the objective function of the discrete formulation. This is solved through mathematical programming (Svanberg 1987).

The layout of the paper is as follows. Section 2 reports fundamentals of governing equations for any linear elastic masonry-like material, focusing on the relevant strain-energy function and introducing a SIMPbased equivalent orthotropic material to model the notension behavior. Section 3 introduces the topology optimization formulation that distributes phases of the equivalent composite according to the stress field found in the no-tension solid. Details on the implemented algorithm are given in Section 3.3. Section 4 presents benchmark examples to assess the effectiveness of the 
proposed method with respect to some established analytical and numerical results found in the literature, while discussing features and performances of the adopted optimization-based procedure. Section 5 concludes the paper, outlining directions of the ongoing research.

\section{Governing equations}

\subsection{Linear elastic masonry-like materials}

A material that does not support tension is considered, meaning that the stress tensor $\sigma_{i j}$ has to be negativesemidefinite, i.e.:

$\sigma_{i j} \in$ Sym $^{-}$,

where $\mathrm{Sym}^{-}$is the closed cone of negative semi-definite symmetric second order tensors. This constraint is equivalent to restricting the principal stresses to be nonpositive.

As usual, the infinitesimal strain tensor is defined as $\varepsilon_{i j}=\frac{1}{2}\left(u_{i, j}+u_{j, i}\right)$, where $\underline{u}$ is the displacement field. The tensor $\varepsilon_{i j}$ is assumed as the sum of an elastic part $\varepsilon_{i j}^{e}$ and a latent one $\varepsilon_{i j}^{c}$, which account for "cracking strains", that means:

$\varepsilon_{i j}=\varepsilon_{i j}^{e}+\varepsilon_{i j}^{c}$.

The elastic part of the strain tensor is related to the stress through the linear relationship:

$\sigma_{i j}=C_{i j h k} \varepsilon_{h k}^{e}$,

where $C_{i j h k}$ is the fourth order positive definite elasticity tensor. The latent part of the strain tensor $\varepsilon_{i j}^{c}$ follows the so-called hypothesis of normality, that reads:

$\sigma_{i j} \varepsilon_{i j}^{c}=0, \quad$ and $\quad \varepsilon_{i j}^{c} \in S y m^{+}$

where $\mathrm{Sym}^{+}$is the closed cone of positive semi-definite symmetric second order tensors. Eqns. (1-4) state that the total strain consists of an elastic part related to negative semi-definite stress $\sigma_{i j}$ through the usual linear operator $C_{i j h k}$, along with a positive semi-definite latent contribution that is orthogonal to $\sigma_{i j}$ to account for "cracking" behavior. The above equations define the so-called linear elastic masonry-like material, see, among the others, Del Piero (1989).

A straightforward example to describe the behavior of structures made of this kind of constrained material is provided by the one-dimensional case of a bar of Young modulus $E$, subject to the axial strain $\varepsilon$. If the bar is shortened, i.e. the total strain $\varepsilon<0$, a negative stress $\sigma=E \varepsilon^{e}$ arises and no cracking is found, that is $\varepsilon^{c}=0$. Stretching the bar for $\varepsilon>0$, no tensile stress is allowed in the structure, that is $\sigma=0$, and the total strain totally turns into "cracking strains", i.e. $\varepsilon^{c}=\varepsilon$ along with $\varepsilon^{e}=0$. Moving to the two-dimensional framework, one has additionally to take into account that Eqn. (4) calls for "cracks" (i.e. a non-negative defined strain) that can only open orthogonally to the nonpositive stress $\sigma_{i j}$, similarly to classically associated plasticity.

As derived in Del Piero (1989), a linear elastic masonry-like material with symmetric elastic tensor $C_{i j h k}$ is hyperelastic and therefore admits a strain-energy density function $\phi\left(\underline{\underline{\varepsilon}}^{e}\right)$ that may be defined as:

$\phi\left(\underline{\underline{\varepsilon}}^{e}\right)=\frac{1}{2} \sigma_{i j}\left(\underline{\underline{\varepsilon}}^{e}\right) \varepsilon_{i j}^{e}$

As for classical non-constrained materials, this function plays the role of a potential for the stress field, i.e.:

$\sigma_{i j}=\frac{\partial \phi\left(\underline{\underline{\varepsilon}}^{e}\right)}{\partial \varepsilon_{i j}^{e}}$

A two-dimensional domain $\Omega$ made of this kind of no-tension material is considered. The boundary of the domain, $\Gamma=\Gamma_{t} \cup \Gamma_{u}$, consists of two different parts: the former is acted upon by tractions $\underline{t}_{0}$, whereas the latter is subjected to prescribed displacements $\underline{u}_{0}$. No body force is supposed to act in the domain from here onwards.

A weak formulation for the elastic equilibrium of the solid may be straightforwardly derived in view of the adoption of a displacement-based finite element method to cope with the discrete form of the problem considered herein. According to Eqns. (5-6), the total potential energy for the linear elastic masonry-like body is defined as:

$\Pi(\underline{v})=\frac{1}{2} \int_{\Omega} \frac{\partial \phi\left(\underline{\underline{\varepsilon}}^{e}(\underline{v})\right)}{\partial \varepsilon_{i j}^{e}(\underline{v})} \varepsilon_{i j}^{e}(\underline{v}) d \Omega-\int_{\Gamma_{t}} \underline{t}_{0} \cdot \underline{v} d \Gamma$,

where $\underline{v} \in H^{1}$ is any kinematically admissible vectorfield such that $\left.\underline{v}\right|_{\Gamma_{u}}=\underline{u}_{0}$. The stationarity condition of the primal variational principle, which is nothing but the usual principle of minimal potential energy, reads: find $\underline{u} \in H^{1}$ such that $\left.\underline{u}\right|_{\Gamma_{u}}=\underline{u}_{0}$ and

$\int_{\Omega} \frac{\partial \phi\left(\underline{\underline{\varepsilon}}^{e}(\underline{u})\right)}{\partial \varepsilon_{i j}^{e}(\underline{u})} \varepsilon_{i j}^{e}(\underline{v}) d \Omega=\int_{\Gamma_{t}} \underline{t}_{0} \cdot \underline{v} d \Gamma$,

$\forall \underline{v} \in H^{1}$ such that $\left.\underline{v}\right|_{\Gamma_{u}}=\underline{u}_{0}$.

Reference is made, among others, to Del Piero (1989), Angelillo (1993), Baratta and Corbi (2005), Baratta and Corbi (2010a), Baratta and Corbi (2010b) and Lucchesi et al. (2000), for extended comments on the features of the above statement and the relevant solutions. It is herein recalled that not all the tractions or displacement boundary conditions correspond to a statically admissible solution of Eqn. (8), i.e. displacements related to an equilibrated stress field that is feasible with respect to the condition of Eqn. (1). For this reason 
only the so-called compatible loads can be investigated to achieve existence of the solution. Additionally, nonuniqueness due to the latent part of the strain has been demonstrated for an extended class of problems, see e.g. Angelillo et al. (2010) and Lucchesi et al. (2000).

\subsection{A strain-energy function for two-dimensional no-tension elastic problems}

The case of isotropic linear elastic masonry-like materials whose elastic behavior is fully described by the Young modulus $E$ and the Poisson's ratio $\nu$ is herein dealt with under plane stress conditions. Let $\sigma_{I}$ and $\sigma_{I I}$ be the eigenvalues of the stress tensor $\sigma_{i j}(\chi)$ computed at any point $\chi \in \Omega$, with $\sigma_{I} \leq \sigma_{I I}$.

The behavior of the no-tension solid may be conveniently investigated by dividing $\Omega$ into three subregions such that $\Omega=\Omega_{1} \cup \Omega_{2} \cup \Omega_{3}$ and:

$$
\begin{aligned}
& \Omega_{1}=\chi \in \Omega: \sigma_{I}<0, \sigma_{I I}<0, \\
& \Omega_{2}=\chi \in \Omega: \sigma_{I}<0, \sigma_{I I}=0, \\
& \Omega_{3}=\chi \in \Omega: \sigma_{I}=0 .
\end{aligned}
$$

In sub-region $\Omega_{1}$ the material is subjected to biaxial compression and behaves like a conventional unconstrained isotropic material. In fact, due to Eqns. (2-4) no latent strain is allowed, that is $\varepsilon^{c}=0$, and the total strain fully turns into the elastic strain $\varepsilon=\varepsilon^{e}$.

In $\Omega_{2}$ the material is subjected to uniaxial compression and behaves like an orthotropic material. According to Eqn. (4) some "cracking strain" $\varepsilon^{c} \geq 0$ is allowed in the direction orthogonal to the tensile isostatic line, whereas a fully elastic behavior is found along the direction of the principal compressive strain. It is worth mentioning that one could alternatively define $\Omega_{2}$ as the locus where the trace of the stress tensor is non-positive, i.e. $\sigma_{11}+\sigma_{22}<0$, and its determinant is null, that is $\sigma_{11} \sigma_{22}-\sigma_{12} \sigma_{21}=0$. The latter condition suggests that the sub-region $\Omega_{2}$ is statically determinate, meaning that the equilibrium equations $\sigma_{i j, i}=0$ are enough to compute stresses. In general, the elastic strain descending from Eqn. (3) is not kinematically admissible and "cracking strain" arises to restore compatibility, see e.g. Angelillo et al. (2010).

Finally, in sub-region $\Omega_{3}$ neither stress nor elastic strain is found and the material behaves like a "void phase", allowing for any positive semidefinite "cracking strain".

Exploiting Eqn. (3) and specializing the plane stress constitutive relations in terms of the principal directions, one may easily turn the conditions enforced on the principal stresses $\sigma_{I}$ and $\sigma_{I I}$ in Eqn. (9) into constraints for the eigenvalues of the strain tensor $\varepsilon_{I} \leq \varepsilon_{I I}$.
Stresses and total strains share the same principal directions due to the linear elastic behavior in compression and the normality condition. Recalling that:

$\sigma_{I}=\frac{E}{1-\nu^{2}}\left(\varepsilon_{I}^{e}+\nu \varepsilon_{I I}^{e}\right), \quad \sigma_{I I}=\frac{E}{1-\nu^{2}}\left(\varepsilon_{I I}^{e}+\nu \varepsilon_{I}^{e}\right),(10$

Eqn. (9) may be straightforwardly re-written as:

$\Omega_{1}=\chi \in \Omega: \varepsilon_{I}<0, \varepsilon_{I I}<-\nu \varepsilon_{I}$,

$\Omega_{2}=\chi \in \Omega: \varepsilon_{I}<0, \varepsilon_{I I} \geq-\nu \varepsilon_{I}$,

$\Omega_{3}=\chi \in \Omega: \varepsilon_{I} \geq 0$.

According to Angelillo et al. (2010), the strain-energy function for a linear elastic masonry-like material undergoing plane stress conditions may be straightforward written in terms of the total strain $\varepsilon$ as:

$\phi(\underline{\underline{\varepsilon}})= \begin{cases}\frac{E}{2\left(1-\nu^{2}\right)}\left(\varepsilon_{I}^{2}+\varepsilon_{I I}^{2}+2 \nu \varepsilon_{I} \varepsilon_{I I}\right) & \text { in } \Omega_{1}, \\ \frac{1}{2} E \varepsilon_{I}^{2} & \text { in } \Omega_{2}, \\ 0 & \text { in } \Omega_{3} .\end{cases}$

In the sub-region $\Omega_{1}$, where the material behaves like a linear elastic isotropic material and no "cracking strain" arises, Eqn. (12.1) provides the conventional contribution $1 / 2\left(\sigma_{I} \varepsilon_{I}+\sigma_{I I} \varepsilon_{I I}\right)$, where $\varepsilon_{I}=\varepsilon_{I}^{e}$ and $\varepsilon_{I I}=\varepsilon_{I I}^{e}$. In $\Omega_{2}$, some strain-energy is stored along the compressive direction for $1 / 2 \sigma_{I} \varepsilon_{I}$ with $\varepsilon_{I}=\varepsilon_{I}^{e}$, while no contribution is due to the orthogonal latent strain, see Eqn. (12.2). The "fully cracked" sub-region $\Omega_{3}$ provides null energy in Eqn. (12.3) since no stress does work over the latent strain.

The above rationale may be easily updated to accommodate plane strain conditions: slight modifications of Eqns. (11) and (12) are required.

It is finally remarked that the approach proposed in Angelillo et al. (2010) solves the equilibrium of linear elastic masonry-like solids seeking for the displacement field $\underline{u}$ that minimizes the potential energy $\Pi(\underline{u})$ in Eqn. (1) according to Eqn. (12). Alternatively, this contribution aims at re-formulating the problem of the elastic equilibrium of masonry-like solids as a topology optimization statement, i.e. finding the distribution of a material with suitable stiffness properties that minimizes the strain energy of Eqn. (12). Brief remarks on this topic are given below.

\subsection{A material interpolation law for no-tension solids}

The problem of distributing some linear elastic material over an assigned domain calls for the definition of a suitable interpolation law for the stiffness properties of the material, depending on a minimization unknown that is, in general, its point-wise density (Kang and 


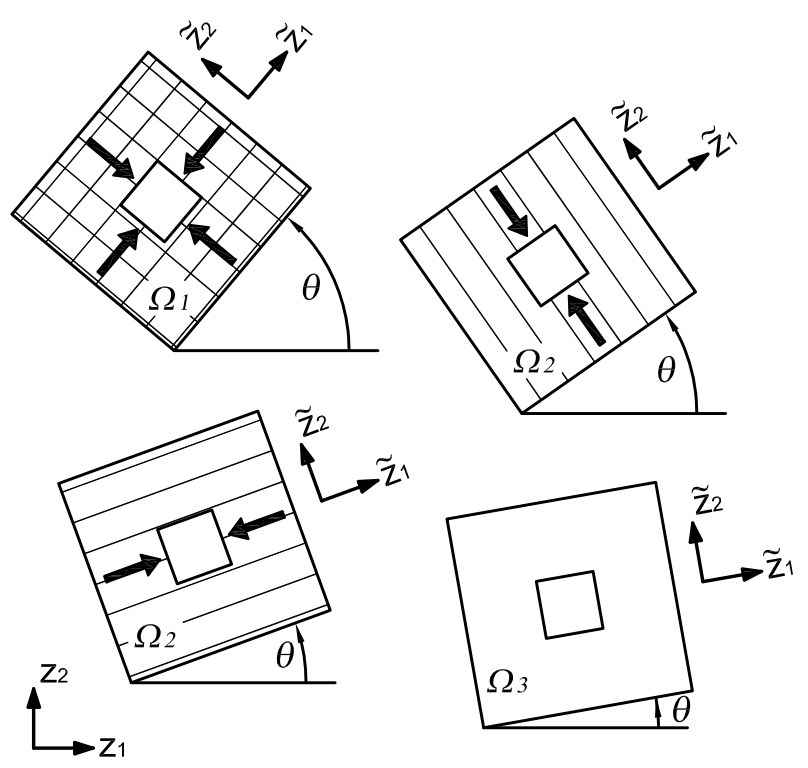

Fig. 1 The adopted material model represented in the general reference of axes $z_{1}$ and $z_{2}$ in $\Omega$ : an orthotropic material phase with symmetry axes $\widetilde{z}_{1}$ and $\widetilde{z}_{2}$ is oriented along the principal stress directions $z_{I}$ and $z_{I I}$ to model the characteristic behavior of a no-tension solid in the three sub-regions $\Omega_{1}, \Omega_{2}$ and $\Omega_{3}$. Lines stand for directions where stiffness of the composite is not negligible.

Wang 2011; Luo et al. 2013). In conventional formulations for the topology optimization of structures made of isotropic material, this is usually done through the adoption of a penalization of the Young modulus in terms of the material density, see e.g. the well-known Solid Isotropic Material with Penalization (SIMP) discussed in Bendsøe and Kikuchi (1988).

Within the framework considered herein, an ad hoc interpolation is needed to model the material behavior of the masonry-like solid through the same analytical form at any point $\chi \in \Omega$. This may be done introducing a suitable set of density unknowns that allow the material behavior to be specialized in each of the subregions $\Omega_{1}, \Omega_{2}$ and $\Omega_{3}$ defined by Eqn. (9) according to the definition of the strain-energy function $\phi(\underline{\underline{\varepsilon}})$ in Eqn. (12).

An orthotropic material whose symmetry axes $\widetilde{z}_{1}$ and $\widetilde{z}_{2}$ are rotated by the angle $\theta$ with respect to the adopted reference frame in $\Omega$, i.e. $z_{1}$ and $z_{2}$, is considered. $\theta$ provides alignment of the symmetry axes of the orthotropic material with respect to the principal direction of stress (and strain) of the masonry-like material $z_{I}$ and $z_{I I}$, see Figure 1. $\widetilde{E}_{1}, \widetilde{E}_{2}$ are the Young moduli of the material (along $\widetilde{z}_{1}$ and $\widetilde{z}_{2}$, respectively), $\widetilde{G}_{12}$ is the shear modulus and $\widetilde{\nu}_{12}, \widetilde{\nu}_{21}$ are the Poisson's ratios with respect to $\widetilde{z}_{1}$ and $\widetilde{z}_{2}$. The equality $\widetilde{\nu}_{12} \widetilde{E}_{1}=\widetilde{\nu}_{21} \widetilde{E}_{2}$ holds.
Let $\rho_{1}(\chi)$ and $\rho_{2}(\chi)$ be two bounded functions in $\Omega$, such that $\rho_{\text {min }} \leq \rho_{1} \leq 1$ and $\rho_{\text {min }} \leq \rho_{2} \leq 1$, respectively, where $0<\rho_{\min }<<1$. The variables $\rho_{1}$ and $\rho_{2}$ are assumed to govern the elastic properties of the orthotropic material along its symmetry axes (i.e. the isostatic stress lines of the masonry-like material) through a generalization of the SIMP model such that:

$$
\begin{aligned}
& \widetilde{E}_{1}=\rho_{1}^{p} E, \quad \widetilde{E}_{2}=\rho_{2}^{p} E, \quad \widetilde{G}_{12}=\rho_{1}^{p} \rho_{2}^{p} \frac{E}{2(1+\nu)}, \\
& \widetilde{\nu}_{12}=\rho_{2}^{p} \nu, \quad \widetilde{\nu}_{21}=\rho_{1}^{p} \nu
\end{aligned}
$$

where $E$ and $\nu$ are the Young modulus and Poisson's ratio of the isotropic masonry-like solid, respectively, while $p>1$ is a penalization parameter that is usually taken equal to 3, see e.g. Bendsøe and Sigmund (1999).

As further detailed, the above interpolation is especially conceived to provide vanishing stiffness along any direction along which a variable gets its minimum value. The constitutive law for the orthotropic phase may be written in general form as:

$\sigma_{i j}=\widehat{C}_{i j h k}\left(\rho_{1}(\chi), \rho_{2}(\chi), \theta(\chi)\right) \varepsilon_{h k}$,

where $\widehat{C}_{i j h k}$ is the fourth order stiffness tensor of the equivalent orthotropic material.

Dealing with a plane problem, Eqn. (14) may be detailed in a more compact form. To this purpose using Voigt notation the cartesian components of the strain tensor $\varepsilon_{i j}$ are re-gathered in the array $\underline{\varepsilon}=\left[\begin{array}{lll}\varepsilon_{11} & \varepsilon_{22} & 2 \varepsilon_{12}\end{array}\right]$ and, analogously, one has $\underline{\sigma}=\left[\sigma_{11} \sigma_{22} \sigma_{12}\right]$ for the components of the stress tensor $\sigma_{i j}$. In general, the symmetry axes of the orthotropic material, $\widetilde{z}_{1}$ and $\widetilde{z}_{2}$, are not the same of the adopted reference $z_{1}$ and $z_{2}$ in $\Omega$. A transformation matrix $\mathbf{T}(\theta)$ is needed to write the constitutive law of the material in the global reference frame $O z_{1} z_{2}$. One has:

$$
\begin{aligned}
\underline{\sigma} & =\mathbf{T}(\theta)^{-1} \mathbf{C}\left(\rho_{1}, \rho_{2}\right) \mathbf{T}(\theta)^{-t} \underline{\varepsilon}, \\
\mathbf{C} & =\frac{1}{1-\widetilde{\nu}_{12} \widetilde{\nu}_{21}}\left[\begin{array}{ccc}
\widetilde{E}_{1} & \widetilde{\nu}_{12} \widetilde{E}_{1} & 0 \\
\widetilde{\nu}_{21} \widetilde{E}_{2} & \widetilde{E}_{2} & 0 \\
0 & 0 & \widetilde{G}_{12}\left(1-\widetilde{\nu}_{12} \widetilde{\nu}_{21}\right)
\end{array}\right], \\
\mathbf{T} & =\left[\begin{array}{ccc}
c^{2} & s^{2} & 2 c s \\
s^{2} & c^{2} & -2 c s \\
-c s & c s & c^{2}-s^{2}
\end{array}\right],
\end{aligned}
$$

where $c=\cos \theta$ and $s=\sin \theta$.

To illustrate the behavior of the orthotropic material introduced in Eqns. (13) to (15) with respect to the no-tension solid referred to in the previous section, a brief discussion is herein reported. Specializing $\mathbf{C}$ in terms of the extremal values of the variables $\rho_{1}$ and $\rho_{2}$, the behavior found in each one of the three sub-regions $\Omega_{1}, \Omega_{2}$ and $\Omega_{3}$ of Eqn. (9) is shown to be recovered by Eqn. (15). For the sake of simplicity, it is assumed that $\theta=0$ and that the cartesian axes are principal 
for the stress and strain fields, i.e. $z_{1}=\widetilde{z}_{1}=z_{I}$ and $z_{2}=\widetilde{z}_{2}=z_{I I}$.

First the case $\rho_{1}=\rho_{2}=1$ is considered. The matrix $\mathbf{C}$ in Eqn. (15) is simply:

$\mathbf{C}\left(\rho_{1}=\rho_{2}=1\right)=\frac{1}{1-\nu^{2}}\left[\begin{array}{ccc}E & \nu E & 0 \\ \nu E & E & 0 \\ 0 & 0 & \frac{E(1-\nu)}{2}\end{array}\right]$,

i.e. the stiffness matrix for an isotropic material undergoing plane stress conditions. According to the above assumption, the strain energy related to Eqn. (16) is the same found in $\Omega_{1}$, see Eqn. (12.1).

It is now assumed that $\rho_{1}=1$ and $\rho_{2}=\rho_{\text {min }}$. In Eqn. (15), the term $\mathbf{C}_{11}$ turns out to be $E$, whereas the other entries are negligible. In this case, a principal stress is recovered along the direction $z_{1}=\widetilde{z}_{1}$ that does work over the relevant strain and provides the same strain energy function previously found in Eqn. (12.2), i.e. the contribution of sub-region $\Omega_{2}$ which experiences "cracking strain" along $z_{2}=\widetilde{z}_{2}$. The same applies if $\rho_{1}=\rho_{\min }$ and $\rho_{2}=1$, meaning that the active principal direction is $z_{2}=\widetilde{z}_{2}$ whereas the axis related to some latent strain is $z_{1}=\widetilde{z}_{1}$.

Finally, for $\rho_{1}=\rho_{2}=\rho_{\text {min }}$ one finds a "void phase" where all the entries of $\mathbf{C}$ are nearly vanishing. A negligible amount of strain energy is stored, see Eqn. (12.2) referring to the "fully cracked" sub-region $\Omega_{3}$.

The above discussion leads to the conclusion that the constitutive law in Eqns. (13) to (15) are able to capture the behavior of linear elastic masonry-like solids described by Eqn. (12), provided that the symmetry axes of the composite phase $\widetilde{z}_{1}, \widetilde{z}_{2}$ matches the principal stress (and strain) directions $z_{I}, z_{I I}$, i.e.:

$\widetilde{z}_{1}=z_{I}, \quad \widetilde{z}_{2}=z_{I I}$

and that the variables $\rho_{1}$ and $\rho_{2}$ obey the following conditions:

$\rho_{1}=\rho_{2}=1, \quad \forall \chi \in \Omega_{1}$,

$\rho_{1}=1, \rho_{2}=\rho_{\text {min }}$, or $\rho_{1}=\rho_{\text {min }}, \rho_{2}=1 \quad \forall \chi \in \Omega_{2},(18)$

$\rho_{1}=\rho_{2}=\rho_{\min } \quad \forall \chi \in \Omega_{3}$.

The above set of equations simply means that the behavior of the equivalent orthotropic material matches that of the masonry-like solid if the variables $\rho_{1}$ or $\rho_{2}$ attain unitary values in case of any compressive stress arising along the relevant principal direction and vanishing values elsewhere. In other words, a penalization of the stiffness constants of the material is required along the direction of any potential tensile stress, see also Eqns. (9).

It is worth remarking that the adoption of a lower bound $\rho_{\min }>0$ allows the energy of the orthotropic material defined in Eqn. (15) to remain positive definite independently of the values of $\rho_{1}, \rho_{2}$ and $\theta$.

A similar discussion holds in case of plane strain conditions.

\section{The topology optimization problem}

\subsection{Introductory remarks}

A formulation of topology optimization for maximum stiffness design finds the distribution of elastic material that minimizes the work of the external load at equilibrium, see e.g. Bendsøe and Kikuchi (1988). Due to Clapeyron theorem, this is equivalent to minimizing the so-called structural compliance $\mathcal{C}$, which is twice the value of the elastic strain energy at equilibrium, i.e. $\mathcal{C}=2 \phi(\underline{\underline{\varepsilon}}(\underline{u}))$. Denoting by $\rho$ the optimization variable that is sought within a suitable set $\rho_{a d}$ of the admissible material densities, this problem may be framed into the following classical statement:

$\begin{cases}\min _{\rho \in \rho_{a d}} \mathcal{C} \\ \text { s.t. } & \frac{\partial \Pi(\underline{v})}{\partial \underline{v}}=0, \quad \underline{v} \in \underline{v}_{a d} \\ & \int_{\Omega}^{\rho} \rho d \Omega=V_{f},\end{cases}$

where Eqn. (19.2) enforces the elastic equilibrium through the principle of stationary potential energy within the class of the kinematically admissible displacements $\underline{v}_{a d}$, see Eqn. (8), whereas Eqn. (19.3) prescribes the volume fraction $0<V_{f} \leq \int_{\Omega} d \Omega$ as the limited amount of material that is available for the optimal design.

According e.g. to Eschenauer and Olhoff (2001), it is straightforward to show that $\Pi(\underline{u})=-\mathcal{C} / 2$, meaning that Eqn. (19) may be re-written as the following alternative statement:

$\left\{\begin{array}{lr}\max _{\rho \in \rho_{a d}} & \min _{\underline{v} \in \underline{v}_{a d}} \Pi(\underline{v}) \\ \text { s.t. } & \int_{\Omega} \rho d \Omega=V_{f} .\end{array}\right.$

It is worth remarking that, for $V_{f}=\int_{\Omega} d \Omega$, the above form reduces to the minimization of the potential energy that is fully equivalent to the unconstrained version of the compliance minimization problem of Eqn. (19). This suggests that the equilibrium of linear elastic masonry-like solids may be alternatively solved through a formulation of topology optimization that properly distributes the orthotropic material defined in Section 2.3 in each one of the sub-regions $\Omega_{1}, \Omega_{2}$ and $\Omega_{3}$ introduced in Section 2.2, in order to minimize the compliance.

Using Eqns. (14) and (15), the weak formulation of the equilibrium of linear elastic masonry-like solids in 
Eqn. (8) may be re--written as follows: find $\underline{u} \in H^{1}$ such that $\left.\underline{u}\right|_{\Gamma_{u}}=\underline{u}_{0}$ and

$$
\begin{aligned}
& \int_{\Omega}\left(\mathbf{T}(\theta)^{-1} \mathbf{C}\left(\rho_{1}, \rho_{2}\right) \mathbf{T}(\theta)^{-t}\right) \underline{\varepsilon}(\underline{u}) \underline{\varepsilon}(\underline{v}) d \Omega \\
& =\int_{\Gamma_{t}} \underline{t}_{0} \cdot \underline{v} d \Gamma,
\end{aligned}
$$

$\forall \underline{v} \in H^{1}$, provided that Eqns. (17) and (18) are satisfied. The above form allows dealing with a no-tension solid as a suitable orthotropic material that exhibits negligible stiffness along principal direction where any potential tensile stress may arise. Contributions to the strain energy are provided along compressive directions where no inelastic strain occurs, see the isotropic subregion $\Omega_{1}$ and the orthotropic sub-region $\Omega_{2}$ along the axis of full density. "Cracking strain" is captured by Eqn. (21) as the latent strain arising along tensile directions to restore kinematics, see the "void phase" $\Omega_{3}$ and the orthotropic sub-region $\Omega_{2}$ along the axis of minimum density.

The discretization of the primal problem of Eqn. (21) may be straightforwardly accomplished through the introduction of the approximated solution field $\underline{u}_{h}$ and test field $\underline{v}_{h}$ that both descend from the adoption of quadrangular isoparametric elements with bi-linear displacement shape functions. A piecewise constant approximation is adopted for $\rho_{1}, \rho_{2}$ and $\theta$, see e.g. Bendsøe and Sigmund (2003) and Bruggi and Taliercio (2013). Eqn. (21) reduces to the following matrix form:

$\mathbf{K}\left(\mathbf{x}_{\mathbf{1}}, \mathbf{x}_{\mathbf{2}}, \mathbf{t}\right) \mathbf{U}=\sum_{e=1}^{N} \mathbf{K}_{e}\left(x_{1 e}, x_{2 e}, t_{e}\right) \mathbf{U}=\mathbf{F}$,

where $\mathbf{K}$ is the global stiffness matrix of the equivalent orthotropic material, depending on the two arrays of element densities $\mathbf{x}_{\mathbf{1}}, \mathbf{x}_{\mathbf{2}}$ and on the array containing the orientation of the symmetry axes of the material in each finite element t. $\mathbf{U}$ stands for the generalized displacement vector, while $\mathbf{F}$ is the load vector. Because of the adopted piecewise constant discretization of $\rho_{1}, \rho_{2}$ and $\theta$, the global stiffness matrix may be straightforwardly assembled from the $N$ element-wise contributions $\mathbf{K}_{e}$, where $N$ is the number of finite elements in the mesh. $x_{1 e}, x_{2 e}$ and $t_{e}$ are the $e^{-}$-th component of the density vectors $\mathbf{x}_{\mathbf{1}}, \mathbf{x}_{\mathbf{2}}$ and the orientation vector $\mathbf{t}$, respectively. After the solution of Eqn. (22) the discrete form of the compliance is generally recovered through:

$\mathcal{C}=\sum_{e=1}^{N} \mathbf{U}_{e}^{T} \mathbf{K}_{e}\left(x_{1 e}, x_{2 e}, t_{e}\right) \mathbf{U}_{e}$,

where $\mathbf{U}_{e}$ is the displacement vector of the $e$-th element.

In view of the detection of the three sub-regions $\Omega_{1}, \Omega_{2}$ and $\Omega_{3}$ depending on the value of the principal stresses in $\Omega$, see Eqn. (9), some post-processing is needed on the displacement vector $\mathbf{U}_{e}$ to implement Eqn. (15). At any selected point of the $e$-th finite element, e.g. the centroid, one may introduce the vectorial form $\underline{\sigma}_{e}=\mathbf{M}_{e}\left(x_{1 e}, x_{2 e}, t_{e}\right) \mathbf{U}_{e}$, being $\underline{\sigma}_{e}$ the array of

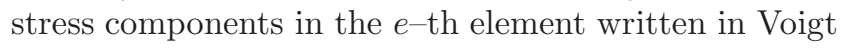
notation, while $\mathbf{M}_{e}$ is its "stress matrix". Eventually, one can compute the principal stresses in the $e$-th finite element, that will be denoted as $\sigma_{e, I}$ and $\sigma_{e, I I}$.

The proposed approach is based on the adoption of conventional four-node displacement-based finite elements but it could be straightforwardly extended to any enhanced discretization to improve the evaluation of the stress regime, see e.g. mixed and truly-mixed finite elements implemented in Bruggi (2008).

\subsection{Problem formulation}

According to the rationale developed above, the equilibrium of linear elastic masonry-like solids may be formulated as the following problem of topology optimization involving orthotropic materials:

$$
\begin{cases}\min _{x_{1 e}, x_{2 e}} & \mathcal{C}=\sum_{e=1}^{N} \mathbf{U}_{e}^{T} \mathbf{K}_{e}\left(x_{1 e}, x_{2 e}, t_{e}\right) \mathbf{U}_{e} \\ \text { s.t. } & \sum_{e=1}^{N} \mathbf{K}_{e}\left(x_{1 e}, x_{2 e}, t_{e}\right) \mathbf{U}=\mathbf{F} \\ & t_{e} \mid \widetilde{z}_{1}=z_{I} \text { and } \widetilde{z}_{2}=z_{I I} \\ & x_{1 e}, x_{2 e} \mid \sigma_{e, I} \leq 0 \text { and } \sigma_{e, I I} \leq 0 \\ & \rho_{\min } \leq x_{1 e}, x_{2 e} \leq 1 .\end{cases}
$$

In the above statement, the objective function is the structural compliance in Eqn. (23) that is computed over the $N$ elements into which the body was discretized, while $x_{1 e}$ and $x_{2 e}$ represent the material density along the symmetry axes of the composite, i.e. $\widetilde{z}_{1}$ and $\widetilde{z}_{2}$ respectively, in the $e$-th finite element. As detailed in Section 2.3, each one of the minimization variables has the same range of variation that is defined by Eqn. (24.5).

Eqn. (24.2) enforces the elastic equilibrium stated by Eqn. (21) in discrete form, see Eqn. (22). Eqn. (24.3) prescribes the alignment of the symmetry axes of the composite $\widetilde{z}_{1}$ and $\widetilde{z}_{2}$ with respect to the principal stress directions $z_{I}$ and $z_{I I}$. This is performed all over the finite element mesh through the orientation array with entries $t_{e}$. Eqns. (24.4) enforce a no-tension stress regime in each finite element, combining Eqns. (9) and (18) to achieve a full match between the behavior of the orthotropic material of Section 2.3 and the target masonrylike solid of Section 2.2 within each of the sub-regions $\Omega_{1}, \Omega_{2}$ and $\Omega_{3}$.

Eqn. (24) formulates the elastic equilibrium of masonry-like solids as a topology optimization problem, thus providing an alternative approach to the non-linear finite element analysis of structures made of no-tension 
material, see Section 1. Whereas many conventional approaches deal with the inherent non-linearity of the problem through step-wise incremental analysis that move from an initial compressive-only stress state, the proposed energy-based method is able to solve the effect of compatible loads within a single optimization run. Unlike the approach in Angelillo et al. (2010), where the displacement field is set as minimization unknown, Eqn. (24) is a topology optimization problem that will be solved through the algorithm presented next.

\subsection{Problem implementation}

As discussed in Section 2.3, a key issue of the procedure is the penalization of the stiffness constants of the material along the direction of any potential tensile stress.

A similar issue arises when dealing with the optimal design of truss-like structures exhibiting unilateral behavior in tension or compression. In the approach proposed in Bruggi and Duysinx (2013), a set of stress constraints is embedded within an energy-based optimization procedure to avoid ties in the optimal layout, while sequential convex programming is adopted to solve the multi-constrained problem. The stiffness penalization is induced through enforcements that control the stress field all over the domain, while an isotropic modeling of the domain is retained to cope with a two-dimensional discretization of truss-like structures.

An alternative method has been proposed in Ananiev (2005) based on a standard minimum compliance formulation for isotropic materials that implements an efficient modification of the projected gradient method to cope with tension-only structures. At each iteration, the density variables are updated according to a modified sensitivity that penalizes the energy contributions deriving from any compressive stress found in the analysis of the isotropic medium. This is shown to provide fast and efficient convergence towards optimal trusslike structures that are fully feasible with respect to the assumed unilateral behavior of the material. A similar approach is implemented in Cai (2011), where a so-called effective strain-energy density is introduced as a modified objective function for the minimum compliance problem. This approach adopts the sign of the principal stress as a penalization coefficient to be applied to any energy contribution that is computed along the relevant principal stress direction. Reference is also made to Querin et al. (2010) for the adoption of stiffness replacement techniques and optimality criteria based on modified stress fields to perform the topology optimization of truss-like continua with different material properties in tension and compression.
The above referenced approaches suggest the adoption of penalization techniques to get rid of tensile stresses by means of limited modifications with respect to the conventional and efficient implementation of the minimum compliance problem, see e.g. Andreassen et al. (2011). The formulation in Eqn. (24) is solved through the adoption of mathematical programming, herein the well-known Method of Moving Asymptotes by Svanberg (1987), that is endowed with objective function and sensitivities computed through penalization techniques extended to the orthotropic framework. Optimality criteria may be alternatively adopted, see e.g. Bendsøe and Sigmund (2003).

The initial guess $x_{1 e}=x_{2 e}=0.5$ is chosen to start the optimization procedure and the orientations $t_{e}$ are assumed to be such that the symmetry axes of the equivalent orthotropic medium $\widetilde{z}_{1}$ and $\widetilde{z}_{2}$ are aligned with the principal stress directions of the isotropic material $z_{I}$ and $z_{I I}$. At each iteration of the minimizer, the following procedure is implemented:

Step 1. A structural analysis is performed according to Eqn. (22) for the current values of the minimization unknowns $x_{1 e}$ and $x_{2 e}$, along with the orientations $t_{e}$. The principal stress directions $z_{I}$ and $z_{I I}$ are computed and each parameter $t_{e}$ is updated in order to provide alignment of the symmetry axes of the composite $\widetilde{z}_{1}$ and $\widetilde{z}_{2}$ with respect to $z_{I}$ and $z_{I I}$, see Eqn. (24.3). The full step is repeated until negligible changes are found for $t_{e}$, in general no more than twice. One ends with $\widetilde{\sigma}_{e, 1}=\sigma_{e, I}$ and $\tilde{\sigma}_{e, 2}=\sigma_{e, I I}$, where $\widetilde{\sigma}_{e, 1}$ and $\widetilde{\sigma}_{e, 2}$ are the normal stresses computed along each one of the symmetry axes of the composite.

Step 2. The densities $\widehat{x}_{1 e}$ and $\widehat{x}_{2 e}$ are introduced for a straightforward computation of a reduced compliance $\widehat{\mathcal{C}}$ where the terms related to principal tensile stresses are penalized, along with its sensitivity information. For $i=1,2$ one has:

$\left\{\begin{array}{l}\widehat{x}_{i e}=x_{i e}, \text { if } \widetilde{\sigma}_{e, i} \leq 0, \\ \widehat{x}_{i e}=k x_{i e}, \text { otherwise, }\end{array}\right.$

where $k \in] 0,1$ [ is a penalization parameter that is herein assumed equal to 0.5, see Ananiev (2005). According to Eqn. (23) the reduced compliance may be computed without any additional computation as:

$\widehat{\mathcal{C}}=\sum_{e=1}^{N} \mathbf{U}_{e}^{T} \mathbf{K}_{e}\left(\widehat{x}_{1 e}, \widehat{x}_{2 e}, t_{e}\right) \mathbf{U}_{e}$.

The sensitivity of the objective function $\mathcal{C}$ with respect to the density unknowns of the $j$-th element may be evaluated through the adjoint method, see e.g. Bendsøe and Sigmund (2003) and Bendsøe et 
al. (1985). For the reduced objective function $\widehat{\mathcal{C}}$ one has:

$$
\frac{\partial \widehat{\mathcal{C}}}{\partial x_{i j}}=\mathbf{U}_{j}^{T} \frac{\partial}{\partial \widehat{x}_{i j}} \mathbf{K}_{j}\left(\widehat{x}_{1 j}, \widehat{x}_{2 j}, t_{e}\right) \mathbf{U}_{j}
$$

where $i=1,2$. Except for the density replacement operated in Eqn. (25), the above equations are nothing but an extension to orthotropic media of conventional computations that can be efficiently implemented in codes for topology optimization.

Step 3. The reduced compliance and its related sensitivities are passed to the minimizer that searches for a new updated set of variables $x_{1 e}$ and $x_{2 e}$, moving from the values $\widehat{x}_{1 e}$ and $\widehat{x}_{2 e}$.

Step 1 to 3 are repeated until convergence is found, i.e. any change in the values of the minimization unknowns between two subsequent iteration is less than a given tolerance (e.g. $10^{-3}$ ).

It is worth remarking that the algorithm presented above, with a few modifications, is able to deal with the topology optimization of no-tension bodies for a prescribed amount of material $V_{f} \leq \int_{\Omega} d \Omega$, see Eqn. (19). In this case the optimizer is additionally endowed with information on the volume constraint and its sensitivities.

Since the statement in Eqn. (24) is equivalent to an analysis problem, no numerical instability that is usually encountered in volume-constrained minimum compliance problems is expected, e.g. mesh dependence or checkerboard effects described e.g. in Sigmund and Petersson (1998). These numerical instabilities may arise if $V_{f}<\int_{\Omega} d \Omega$, calling for suitable remedies such the adoption of filtering techniques, see among the others Bourdin (2001), Bruns and Tortorelli (2001), Sigmund (2007), Guest et al. (2004), Guest (2009).

\section{Numerical simulations}

Numerical investigations are presented to assess the method proposed in Sections 3.2 and 3.3. Examples 1 and 2 show the main features of the proposed computational approach when dealing with the approximation of discontinuous stress and displacement fields. Results are compared with exact analytical solutions available in the literature. Examples 3 and 4 investigate the adoption of the proposed algorithm to cope with the evaluation of the limit load of benchmark structures previously addressed in the numerical literature on notension solids.

It must be remarked that the adopted computational tool descends from an optimization formulation that is conceived to solve the structure for any compatible load. As shown hereafter, no incremental analysis

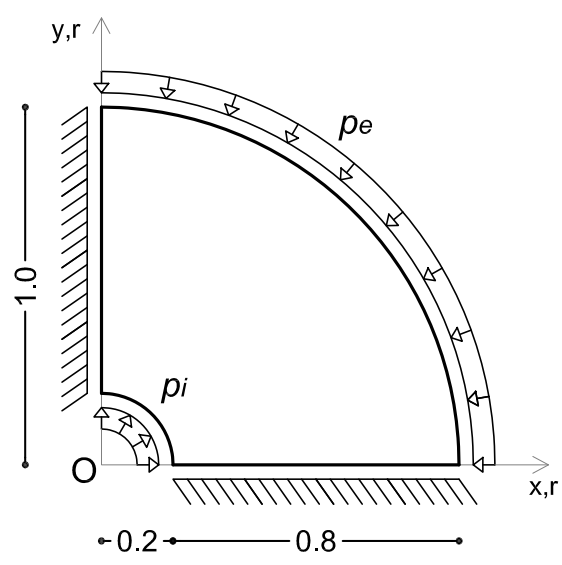

Fig. 2 Geometry and boundary conditions for the annulus of example 1. Dimensions in $m$, unitary thickness.

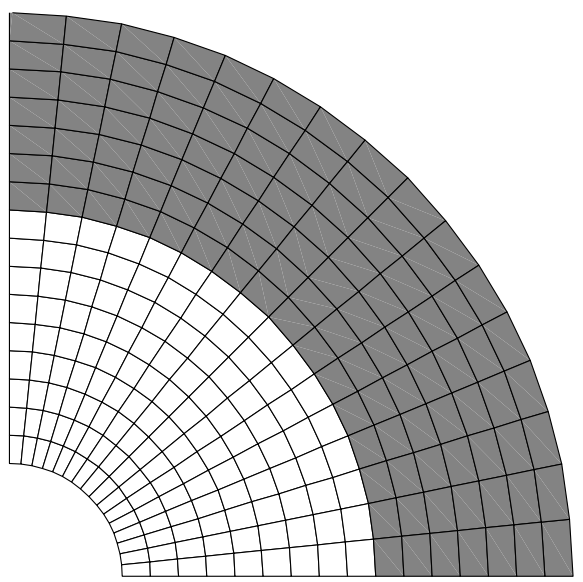

Fig. 3 Example 1. "Optimal distribution of material" for the analysis of the annulus: black stands for linear isotropic material; white stands for non-linear orthotropic material (mesh with 16x16 elements).

neither enforcement of compression-only starting guess are needed to achieve the expected solution.

\subsection{Example 1. Annulus subject to internal-external} pressure

The portion of the annulus represented in Figure 2 is firstly analyzed, as originally investigated by Cuomo and Ventura (2000). Elastic properties of the material are as follows: $E=10,000 \mathrm{MPa}, \nu=0.1$. The domain is loaded by an external pressure $p_{e}=1.00 \mathrm{MPa}$ and an internal one $p_{i}=0.22 \mathrm{MPa}$. Three different meshes are considered, see e.g. Figure 3. All of them share 16 elements in the hoop direction and 8,16 or 32 in the radial direction. Symmetry conditions have been exploited to avoid the modeling of the whole annulus. 


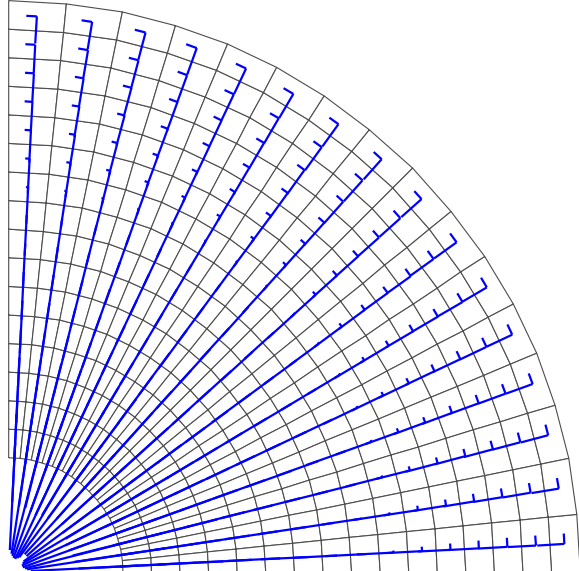

Fig. 4 Example 1. Principal compressive stress in the annulus (mesh with 16x16 elements).

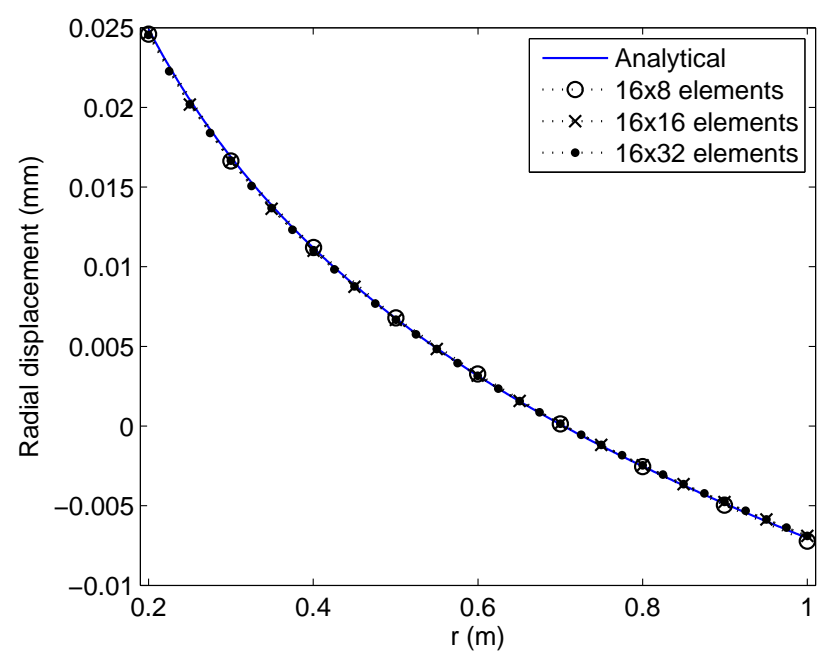

Fig. 5 Example 1. Radial displacement in the annulus: numerical results vs. analytical solution.

Analytical solutions for this problem are available in Bennati and Padovani (1992), both in the case of linear elastic material and as no-tension material. According to the exact solution found for masonry-like materials, a circle with radius $r_{0}=641.7 \mathrm{~mm}$ divides the linear region (where the material is isotropic) from the nonlinear one (where the solid behaves as an orthotropic composite). It must be remarked that the radius referred above differs from that divides the compressive zone from the tensile region under the conventional assumption of linear elastic material.

Figure 3 presents the "optimal distribution of material" achieved by the proposed numerical procedure for this benchmark. As detailed in Section 3.2, the adopted numerical scheme is an optimization method that distributes a phase of orthotropic material through the

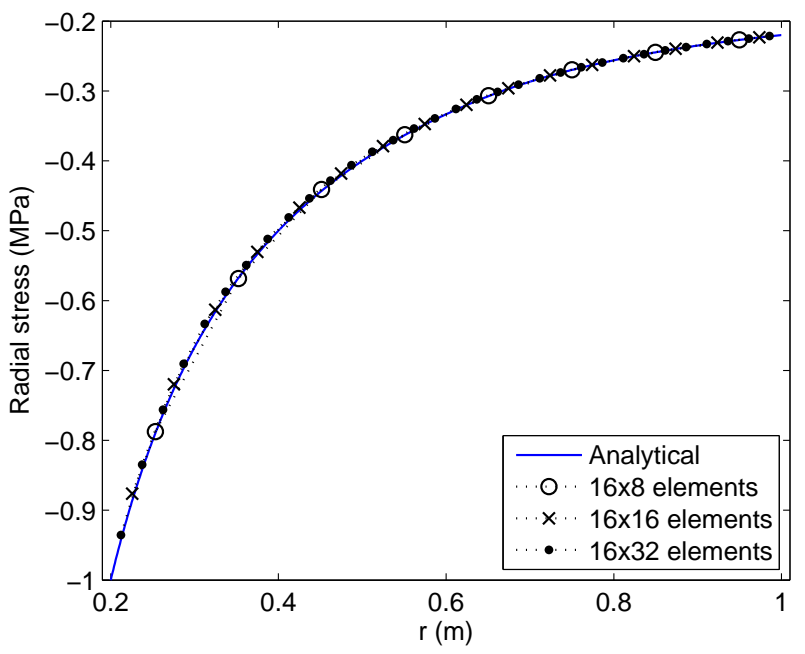

(a)

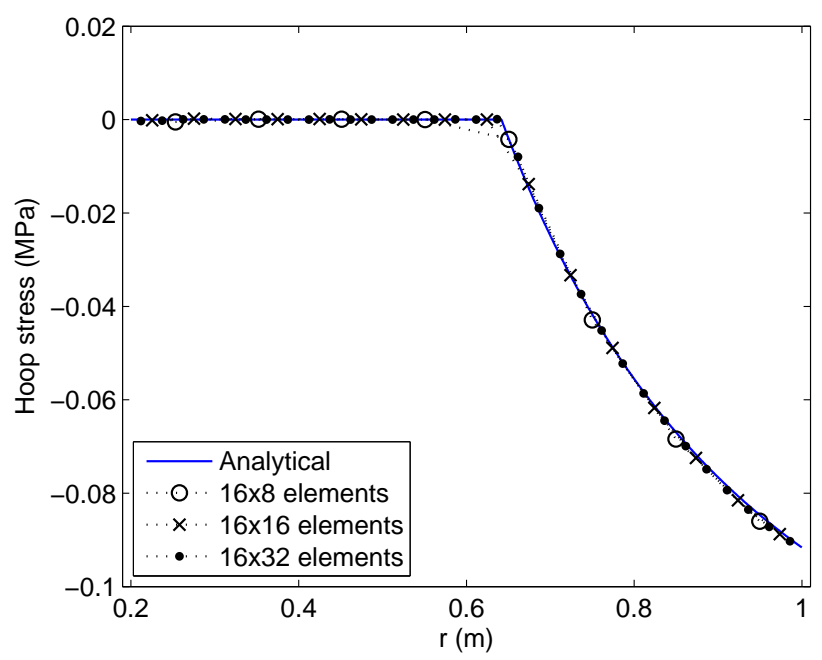

(b)

Fig. 6 Example 1. Radial (a) and hoop (b) stress in the annulus: numerical results vs. analytical solution.

search for the optimal sets of the discrete density variables $x_{1 e}$ and $x_{2 e}$. Black elements stand for linear isotropic material, i.e. $x_{1 e}=x_{2 e}=1$, whereas white zones detect regions exhibiting a non-linear orthotropic behavior, herein for $x_{1 e}=1$ along with $x_{2 e}=\rho_{\min }$. The topological plot of Figure 3 is an "optimal design" in the sense that it is the result of a problem of topology optimization; indeed, it represents the distribution of linear/non-linear material that minimizes the strainenergy of the no-tension body i.e. solves the equilibrium of the structure.

At each step of the optimization procedure a finite element analysis is required to compute the displacementbased solution of the state equation for the current distribution of density variables, see Section 3.3. At con- 


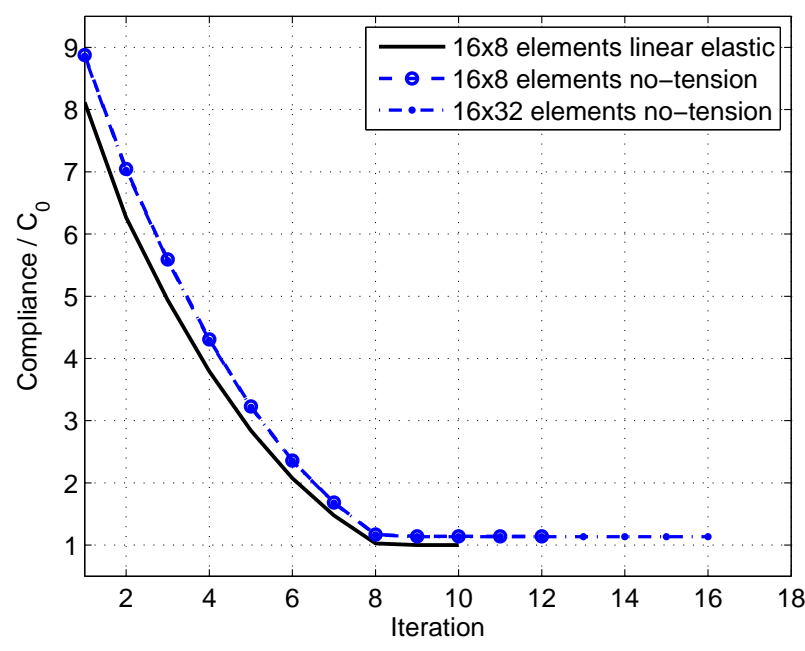

Fig. 7 Example 1. Convergence plots for different discretizations and assumptions on the material behavior.

vergence, i.e. for the "optimal distribution of material" shown in Figure 3, the analysis provides the sought solution for the no-tension structure.

Figure 4 shows the principal stresses computed in the annulus at convergence, at the centroid of each finite element of the mesh, i.e. at the points where the stress field is controlled by the proposed procedure. As expected, compressive principal stresses arise along any radius of the domain and along any hoop direction falling within the linear isotropic region. No hoop stress is found within the non-linear orthotropic zone, meaning that some latent strain arises to account for scattered cracking. Figures 5 to 6 compare the achieved numerical results with the available analytical solution, plotting relevant displacement and stress profiles along the radius of the annulus. Displacements are computed at the finite element nodes, whereas stresses refer to the centroid of each element. The three discretizations adopted in this numerical investigation provide approximately the same results, which are in full agreement with the exact analytical solution. In particular, it may be noticed that the discontinuous profile of the hoop stress is captured by the achieved numerical solutions, irrespective of the refinement of the mesh. In fact, all the points plotted in Figure 6(b) lie over the nonsmooth line representing the analytical solution. The adoption of finer meshes allows increasing the accuracy in the approximation of $r_{0}$.

Finally, Figure 7 provides information about the convergence of the implemented optimization procedure. The curves in the diagram represent history plots of the non-dimensional objective function, i.e. the ratio of the structural compliance to the strain energy com- puted for the initial guess, i.e. $x_{1 e}=x_{2 e}=0.5$ along with $\widetilde{z}_{1}=z_{I}$ and $\widetilde{z}_{2}=z_{I I}$ (see Section 3.3). Two curves are reported concerning the analysis of the annulus under the assumption of a no-tension behavior, which are the convergence plots for the coarsest and the finest meshes adopted in the simulations. Both optimization procedures find convergence at the same energy threshold, in agreement with the mesh independence observed in the results of Figures 5 to 6 . A very limited number of iterations is needed to meet the prescribed stopping criterion. It is much lower than that required in conventional formulations for the optimal design of stiff structures where an additional volume constraint is active.

The convergence plot for the coarsest mesh that is analyzed under the assumption of linear elastic material is represented in Figure 7 as well. In this case the optimizer moves from the starting guess adopted for the notension behavior to achieve a trivial solution consisting in full material density all over the domain. Comparing the three curves plotted in the figure one may easily conclude that accounting for the no-tension assumption through the penalization approach presented in Section 3.3 is an efficient choice from the computational point of view. Re-formulating the problem of the analysis of no-tension materials within a topology optimization framework leads to a discrete setting that may be robustly tackled by the proposed algorithm at low computational cost. Additionally, the number of unknowns involved in the considered analysis problem is much lower than that found in conventional approaches of optimal design. This means that minimization algorithms that are especially conceived for large scale optimization are able to solve the herein proposed formulation in a very short time.

\subsection{Example 2. Panels subject to vertical loads}

The rectangular masonry-like panels represented in Figure 8 are acted upon by an external distributed vertical load, i.e. $p=0.1 \mathrm{MPa}$. This investigation has been inspired by that originally performed in Cuomo and Ventura (2000) to assess the capability of the numerical approach proposed therein in capturing the solution for the no-tension plain panel in Figure 8(a). Similarly to Cuomo and Ventura (2000), the Young modulus $E$ is assumed to be equal to $5000 M P a$, whereas the Poisson ratio vanishes. Plane stress conditions are dealt with.

Dealing with the no-tension assumption, the half of the panel that is loaded by the external load behaves independently of the remaining part of the structure. In fact, neglecting strength in tension prevents 

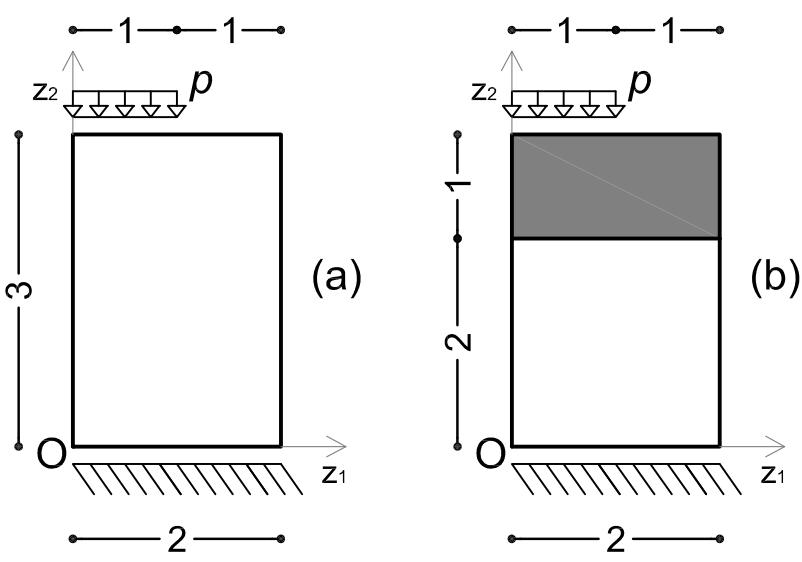

Fig. 8 Geometry and boundary conditions for panels (a) and (b) of example 2. Grey stands for "rigid" elements. Dimensions in $m$, unitary thickness.

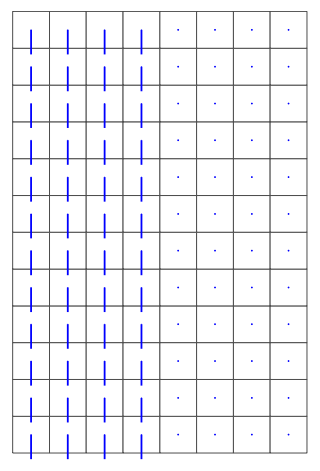

(a)

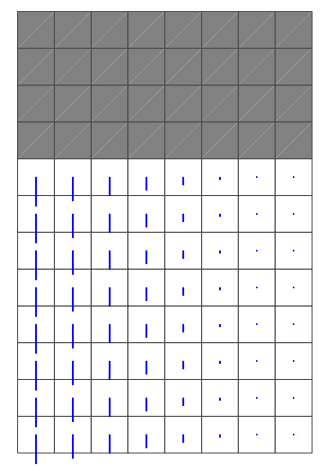

(b)
Fig. 9 Example 2. Principal compressive stress in the panels (a) and (b) of Figure 8 (mesh with 8x12 elements).

any spread of the uniformly distributed pressure beyond the loaded region. Figure 9 (a) shows a plot of the principal compressive stresses found in the panel by the proposed optimization procedure for a discretization of 8 elements along the base and 12 along the height. A pressure diagram as that represented by the solid line in Figure 10(a) is expected along any horizontal section, showing that a discontinuity arises in the stress field for $z_{1}=1 \mathrm{~m}$. Two levels of refinement are investigated to assess mesh independence of the proposed procedure when capturing the outlined pressure profile. As already commented when addressing the results in Figure 6(b), a finer discretization allows for more accuracy in the definition of the stress field next to the discontinuity region, but no remarkable effect is found elsewhere. If an accurate modeling of the stress jump is needed (e.g. to evaluate the size of the linear elastic region), a richer discretization may be implemented within the proposed approach at a slightly increased computational effort.

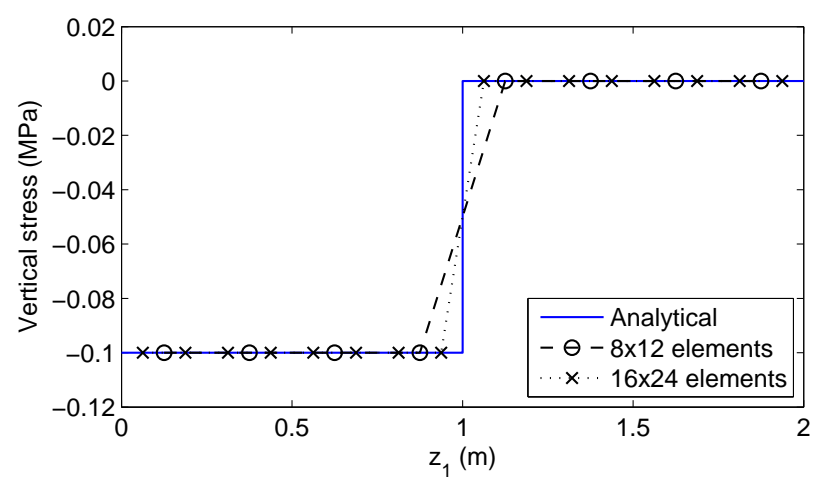

(a)

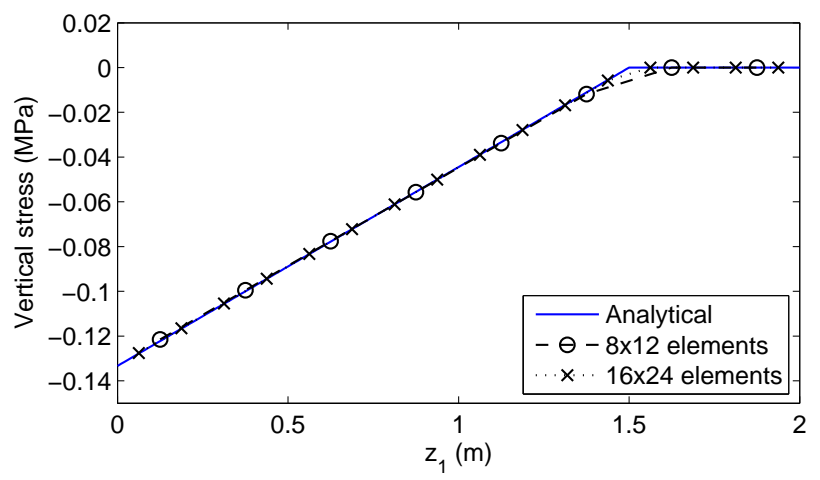

(b)

Fig. 10 Example 2. Vertical stress in the panels (a) and (b) of Figure 8: numerical results vs. analytical solutions.

Well-known concepts applied in the retrofitting of existing masonry-like structures inspire the adoption of tensile-resistant stiff elements, such as reinforced concrete curbs or steel beams, to facilitate the spread of the external pressure beyond the loaded region and to involve a larger portion of the no-tension structural element in the load transfer path. Figure 8(b) shows a variation of the original example investigated in Cuomo and Ventura (2000), where the upper third of the masonrylike panel is replaced by a nearly rigid tensile-resistant structural element whose Young modulus $E_{r}=10000 E$.

As captured by the proposed numerical procedure, a spread of the uniformly distributed load is achieved and the inactive part of the panel significantly reduces with respect to the unreinforced case analyzed above, see Figure 9(b). The "curb" acts as a rigid link that is able to enforce a linear profile to the normal strain along any horizontal section of the masonry-like panel, meaning that linear stresses are expected in the compressive region. The equilibrium of the panel allows computing the vertical stress at any horizontal section: straightforwardly

$\begin{array}{lll}\sigma_{z 2}=\frac{4 p e}{{\overline{z_{1}}}^{2}}\left(z_{1}-\overline{z_{1}}\right) & \text { for } & z_{1} \leq \overline{z_{1}} \\ \sigma_{z 2}=0, & \text { for } & z_{1} \geq \overline{z_{1}}\end{array}$ 


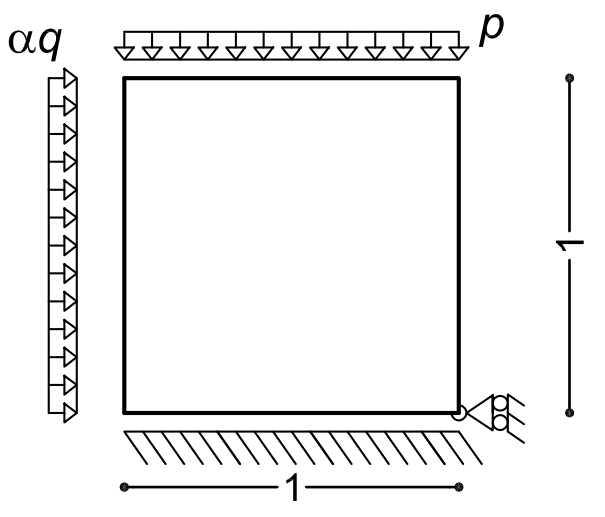

Fig. 11 Geometry and boundary conditions for the panel of example 3. Dimensions in $m$, thickness $0.01 m$.

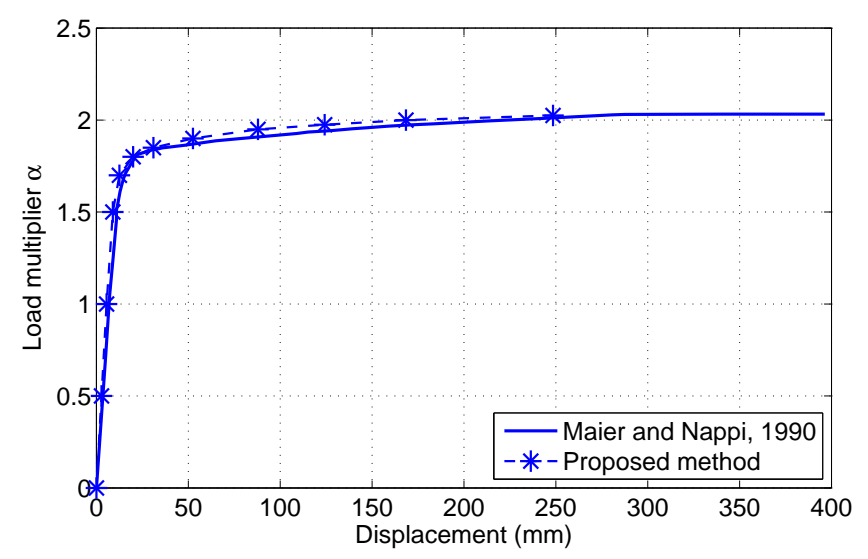

Fig. 12 Example 3. Top right horizontal displacement of the panel vs. load multiplier $\alpha$.

where $e$ is the eccentricity of the load resultant with respect to the axis $z_{2}$, herein $e=0.5 \mathrm{~m}$, whereas $\overline{z_{1}}=$ $3 e=1.5 \mathrm{~m}$ is the abscissa dividing the linear part of the panel from the non-linear one. The solid line in Figure 10(b) shows the analytical solution in Eqn. (28) along with the vertical stress computed by the proposed numerical procedure at the centroid of the elements for two different refinements of the adopted mesh. Both discretizations are able to capture the expected stress profile. Again, a finer mesh allows the exact abscissa that separates the linear region of the panel from the non-linear one to be determined with more accuracy.

\subsection{Example 3. Square panel subject to lateral loads}

As outlined in Section 1, finite element approaches for no-tension materials have been successfully adopted in the last decades as numerical tools to evaluate collapse multipliers for structural elements that are usually handled through methods of limit analysis. Although the

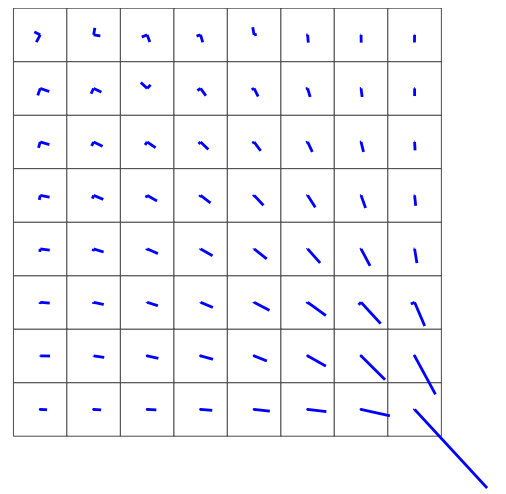

Fig. 13 Example 3. Principal compressive stresses at incipient collapse $(\alpha=2.025)$.

compressive behavior is different, the same no-tension requirement is implemented within both approaches. Computational methods for no-tension materials allow preliminary investigations to be performed accounting for the lack in tensile strength that may be observed in a large class of masonry-like structures, thus providing a valuable tool to assess the safety of historical buildings against collapse under vertical and horizontal loads, see e.g. Maier and Nappi (1990); Cuomo and Ventura (2000); Alfano et al. (2000); Baratta and Corbi (2010a,b).

The square panel sketched in Figure 11 is analyzed, as originally presented in Maier and Nappi (1990) and also investigated, among the others, in Fuschi et al. (1995). The Young modulus is taken equal to $1000 \mathrm{MPa}$, while the Poisson ratio $\nu=0.2$. A vertical dead load $p$ of $18 \mathrm{kN} / \mathrm{m}$ is applied along the top edge of the square wall, whereas a horizontal live load $\alpha q$ acts upon its left edge, where $q=10 \mathrm{kN} / \mathrm{m}$ and $\alpha$ is the load multiplier.

A mesh of $8 x 8=64$ elements is herein adopted with the aim of testing the proposed approach for a number of stress evaluation points similar to that employed in Maier and Nappi (1990) and Fuschi et al. (1995). Figure 12 shows curves representing the top right horizontal displacement of the no-tension block versus the horizonal load multiplier, comparing results achieved by the proposed numerical procedure (dots) with the numerical simulation originally performed in Maier and Nappi (1990) (solid line).

Unlike most of the available computational tools that cope with no-tension materials implementing incremental approaches, the proposed method does not call for any step-by-step analysis that is generally required to handle the load history. Indeed, for each prescribed value of the load multiplier, the optimization setting in Eqn. (24) allows computing the relevant dis- 


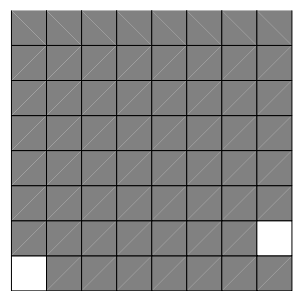

(a)

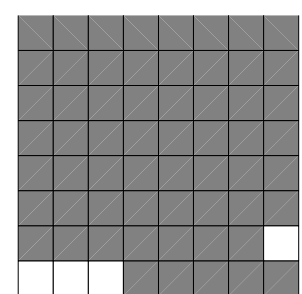

(b)

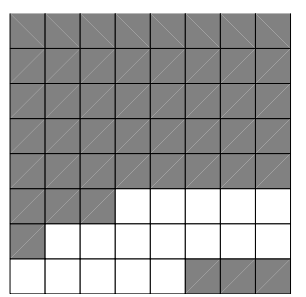

(c)

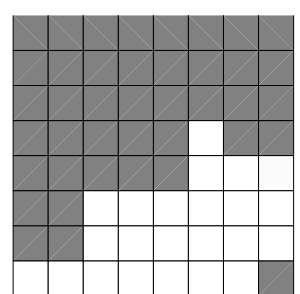

(d)

Fig. 14 Example 3. "Optimal distribution of material" for different values of the load multiplier: $\alpha=1.00$ (a), $\alpha=1.50$ (b), $\alpha=1.80$ (c), $\alpha=1.95$ (d). Black stands for linear isotropic material. White stands for non-linear orthotropic material.

placement of the control point independently of the previous history.

The dotted curve in Figure 12 connects the results achieved by a set of converged optimization runs that have been performed for increasing values of the parameter $\alpha$ to assess the proposed method with respect to the referenced numerical results. A lack of convergence of the proposed algorithm states that no feasible equilibrium solution can be found according to the no-tension assumption, meaning that a collapse of the structure is expected for the considered value of the load multiplier. Figure 13 shows a plot of the principal stresses computed at the last converged simulation, for $\alpha=2.025$. Except for the region next to the ground hinge, the direction of the compressive stresses at the base of the panel is nearly horizontal, meaning that a global collapse of the structure is incipient, see Maier and Nappi (1990).

To have an insight on the non-linear behavior of the panel under increasing lateral loads, Figure 14 collects plots representing the "optimal distribution of material", i.e. the distribution of the minimization unknowns governing the stiffness of the equivalent orthotropic material, for some values of the multiplier $\alpha$. As usual, black regions detect the isotropic fully linear material whereas white elements spot out the sources of nonlinearity that affect the overall stiffness of the panel. In the layouts of Figure 14(a-b), the very few elements exhibiting a non-linear behavior have a minor effect on the compliance of the structure, see Figure 12. Conversely, a dramatic loss in stiffness is found when larger regions become non-linear and the reacting length along the base of the panel reduces to a single element next to the ground hinge, see Figure $14(\mathrm{c}-\mathrm{d})$. It is worth remarking that each one of the analysis represented in Figure 14 is independent of the others.

Some of the incremental approaches proposed in the literature to cope with no-tension materials call for a starting configuration of the external loads for which only compressive stresses arise in the solution, meaning that any non-linear behavior is forbidden at the be-

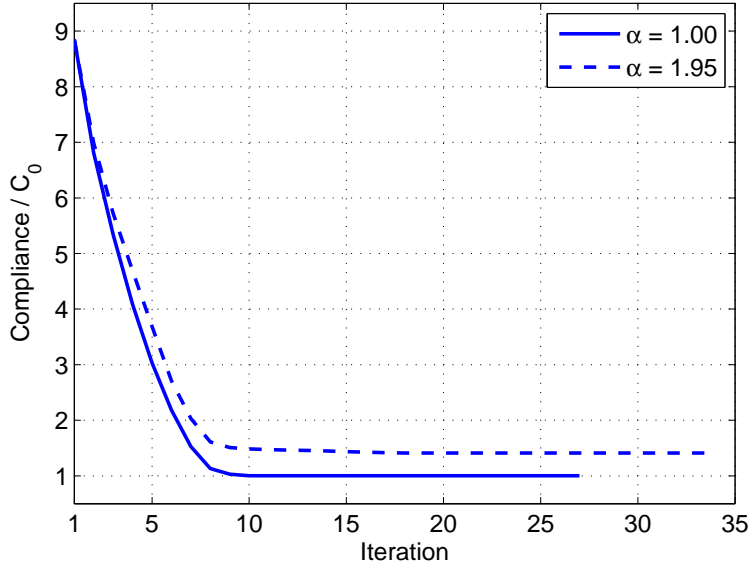

Fig. 15 Example 3. Convergence plot for $\alpha=1.00$ (see Fig. 14a) and $\alpha=1.95$ (see Fig. 14d).

ginning of the load history. Prestressing forces may be added to applied compatible loads to meet this requirement, see e.g. discussions in Fuschi et al. (1995); Alfano et al. (2000). The one-shot optimization procedure in Eqn. (24) does not share this need.

Figure 15 investigates the effect of the amplitude of the non-linear region found in the "optimal distribution of material" with respect to the convergence features of the adopted solving algorithm. To this purpose, the non-dimensional history plots of the structural compliance are compared for two values of the load multiplier, i.e. $\alpha=1.00$ and $\alpha=1.95$. For the former value very few non-linear elements arise in the domain, whereas for the latter nearly half of the panel is found to be non-linear, see Figures 14(a) and 14(d). Smooth curves are reported in both cases. The solution of the more compliant structure requires only a few additional iterations respect to the analysis of the quasi-linear panel. This points out an attractive feature of the implemented procedure, i.e. its very low sensitivity to the number of non-linear finite elements arising in the optimal solution. 


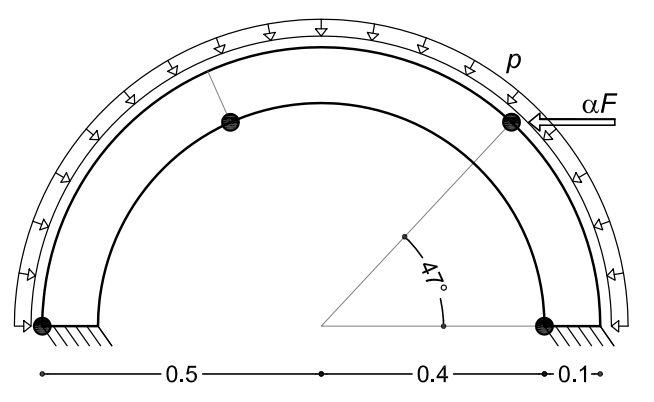

Fig. 16 Geometry, boundary conditions and expected location of hinges in the collapse mechanism for the circular arch of example 4. Dimensions in $m$, thickness $0.1 m$.

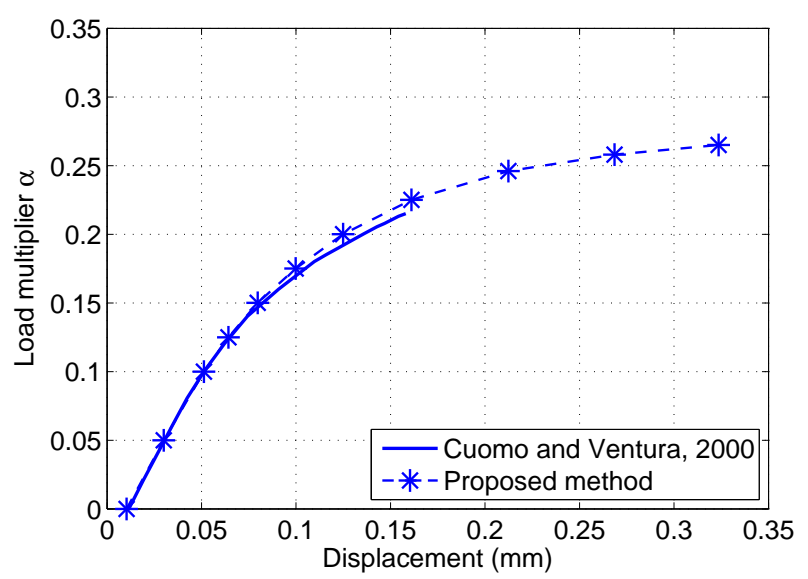

Fig. 17 Example 4. Displacement of the loaded point vs. load multiplier $\alpha$.

\subsection{Example 4. Circular arch subject to lateral force}

The circular arch represented in Figure 16 is finally analyzed. The elastic properties of the material are as follows: $E=10,000 \mathrm{MPa}, \nu=0.1$. A pressure of $0.1 \mathrm{MPa}$ acts along the external perimeter of the arch, while a lateral force $\alpha F$ with $F=1 \mathrm{kN}$ is applied at approximately half of the height of the arch. This structure was originally investigated in Cuomo and Ventura (2000) with the aim of comparing the value of the collapse multiplier $\alpha$ computed through a no-tension complementary energy formulation solved through an augmented Lagrangian iteration Cuomo and Ventura (1998) to the result achieved by a conventional limit analysis. Figure 16 shows the location of the "hinges" that activate the collapse mechanism assumed in the application of the lower bound theorem for an ultimate load multiplier $\alpha_{c}=0.272$, see Cuomo and Ventura (2000).

The solid line in Figure 17 shows a diagram of the horizontal displacement of the point loaded by the lateral force versus the load multiplier $\alpha$, as computed
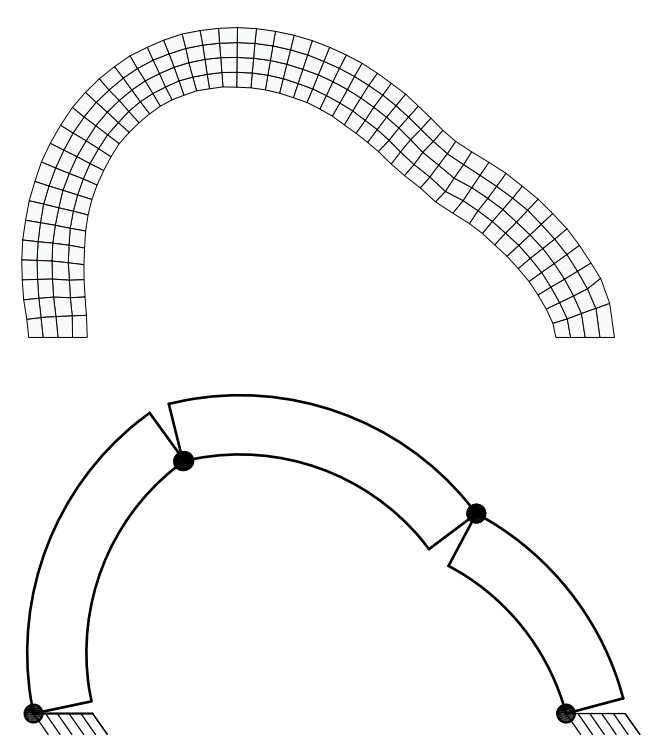

Fig. 18 Example 4. Computed deformed geometry for $\alpha=0.265$ (magnification factor 400) vs. collapse mechanism from limit analysis.

in Cuomo and Ventura (2000) through a discretization adopting 4 elements along the thickness of the arch and 20 in the hoop direction. Following the discussion reported in Alfano et al. (2000), a finer mesh is herein implemented consisting of 48 elements along the hoop direction with the main aim of capturing the behavior of the arch at incipient collapse. A dramatic loss in the overall stiffness should be observed in the diagram of Figure 17, right before the activation of the collapse mechanism.

The dotted line in Figure 17 connects (independent) results achieved by the proposed optimization procedure for increasing values of the parameter $\alpha$, see Example 3. A good agreement is found in the first part of the diagram with respect to the numerical results referenced above. The last value of the load multiplier for which the optimization algorithm finds convergence is $\alpha=0.265$ that approximately matches the value found by limit analysis $\alpha_{c}$. A noticeable decrease in slope of the load-displacement curve is observed approaching this value.

Figure 18 compares the deformed geometry computed by the proposed procedure at incipient collapse with the collapse mechanism assumed in the limit analysis performed in Cuomo and Ventura (2000). Figure 19 provides the map of the principal stresses for the same extreme value of the load multiplier. Most of the structure is acted upon by compressive stresses, whereas a stress relieve is found in a few limited regions of the 


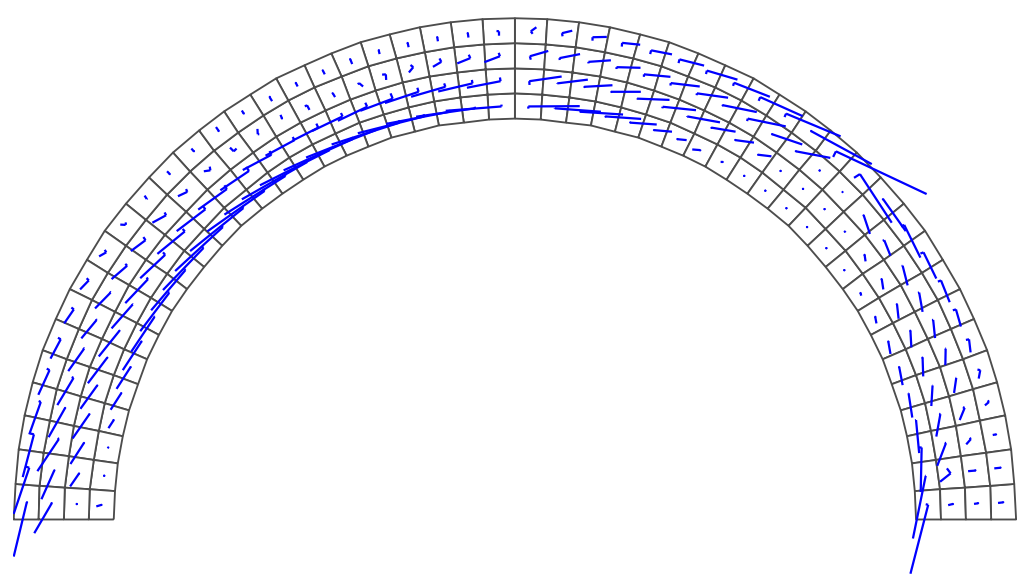

Fig. 19 Example 4. Principal compressive stresses for $\alpha=0.265$.

domain where the arising of some "cracking strain" is expected. These regions are approximately located where the "hinges" of the collapse mechanism of Figure 16 are supposed to activate. Indeed, the theory of no-tension materials allows for a finer description of the stress field leading to the collapse mechanism with respect to limit load analysis that assumes a much simpler stress block in compression. Following e.g. Cuomo and Ventura (2000); Alfano et al. (2000), the above results encourage the adoption of efficient numerical methods based on the no-tension assumption to provide investigations on the behavior of masonry-like structures at incipient collapse.

\section{Conclusions}

A numerical approach has been presented to address the analysis of isotropic no-tension masonry-like solids as a problem of topology optimization. The equilibrium of a two-dimensional no-tension body is found searching for the distribution of an equivalent orthotropic material such that the potential energy is minimized. Two sets of density unknowns are introduced to define an ad hoc interpolation law that controls the compliance of the composite along its symmetry axes, which should be oriented to match the principal stress directions of the no-tension solid. The model recovers the conventional isotropic behavior for biaxial compression, whereas it is able to enforce negligible stiffness along a principal direction where any potential tensile stress may arise. A suitable penalization is adopted to weight the energy contributions involving tensile stresses within the objective function, having the aim of providing an efficient optimization scheme that is solved through mathematical programming.
Whereas most of the computational approaches conceived for no-tension materials resort to incremental analysis following any load history step-by-step, the proposed energy-based method is able to cope with the inherent non-linearity of the problem through a oneshot optimization for any compatible load case. Numerical simulations show that the proposed approach is able to predict analytical and numerical solutions available in the literature for benchmark examples, exhibiting good accuracy even when adopting coarse meshes. The discrete optimization problems that arise when tackling a single structural component are small-scale (if compared with standard topology design settings) and can be solved at a limited computational cost. Additionally, smoothness of convergence is preserved irrespective of the amplitude of the non-linear region that is expected to arise in the solution. This means that the proposed method seems well-suited to the analysis and assessment of real-world masonry-like continues, since topology optimization can efficiently handle large-scale problems involving very fine discretizations, see in particular Andreassen et al. (2011).

The above features suggest the adoption of the proposed approach to provide preliminary investigations on the behavior of masonry-like structures at incipient collapse. Indeed, losses of equilibrium due to the extreme non-linear behavior under collapse loads may be efficiently tackled within the implemented optimization setting. In full agreement with previous experiences of the computational literature, limit loads based on the no-tension assumption have been recovered through the proposed numerical tool, discussing features of the expected collapse mechanisms as well. Indeed, the proposed method seems well-suited also to the investiga- 
tion of local and global collapse mechanisms arising in real-world masonry-like continues.

Extension to the three-dimensional framework is currently under investigation, with the main aim of addressing structural elements and structures having complex geometry, see e.g. vaults and historical buildings. The ongoing research is also focused on the generalization of the proposed approach to account for orthotropy in the linear elastic behavior of masonry under biaxial compression.

\section{Acknowledgement}

The author wishes to thank Prof. Alberto Taliercio for stimulating discussion on the approaches available to the analysis of masonry-like structures and for his constructive comments on the present work.

\section{References}

Alfano G, Rosati L, Valoroso N (2000), A numerical strategy for finite element analysis of no-tension materials. Int J Numer Methods Eng 48(3):317-350

Ananiev S (2005), On equivalence between optimality criteria and projected gradient methods with application to topology optimization problem. Multibody System Dynamics 13(1):2538

Andreassen E, Clausen A, Schevenels M, Lazarov BS, Sigmund O (2011), Efficient topology optimization in MATLAB using 88 lines of code. Struct Multidiscip Optim 43:1-16

Angelillo M (1993), Constitutive relations for no-tension materials. Meccanica 28(3):195-202

Angelillo M, Cardamone L, Fortunato F (2010), A numerical model for masonry-like structures. Journ of Mech of Mater and Struct 5(4):583-615

Baratta A, Corbi O (2005), On variational approaches in NRT continua. Int Journ Solids Structures 42(20):5307-5321

Baratta A, Corbi O (2010), An approach to masonry structural analysis by the no-tension assumption - Part I: Material modeling, theoretical setup, and closed form solutions. Appl Mech Rev 63(4):040802

Baratta A, Corbi O (2010), An approach to masonry structural analysis by the no-tension assumption - Part II: Load singularities, numerical implementation and applications. Appl Mech Rev 63(4):040803

Bendsøe MP, Kikuchi N (1988), Generating optimal topologies in structural design using a homogeneization method. Comp Meth Appl Mech Eng 71:197-224

Bendsøe MP, Olhoff N, Sokolowski J (1985), Sensitivity analysis of problems of elasticity with unilateral constraints. Journ Struct Mech 13(2):201-222

Bendsøe MP, Sigmund O (1999), Material interpolation schemes in topology optimization. Arch Appl Mech 69:635-654

Bendsøe MP, Sigmund O (2003), Topology Optimization - Theory, Methods and Applications. Springer, Berlin

Bennati S, Padovani C (1992), Soluzioni esplicite per problemi di equilibrio di solidi non reagenti a trazione. Proc XI AIMETA National Congress, Solids and Structures Mechanics, Trento, Italy, 73-78
Bourdin B (2001), Filters in topology optimization. Int J Numer Methods Eng 50:2143-2158

Bruggi M (2008), On the solution of the checkerboard problem in mixed-FEM topology optimization. Comput Struct 86(1920):1819-1829

Bruggi M, Duysinx P (2012), Topology optimization for minimum weight with compliance and stress constraints. Struct Multidiscip Optim 46(3):369-384

Bruggi M, Duysinx P (2013), A stress-based approach to the optimal design of structures with unilateral behavior of material or supports. Struct Multidiscip Optim 48(2):311-326

Bruggi M, Taliercio A (2013), Topology optimization of the fiber-reinforcement retrofitting existing structures. Int Journ Solids Struct 50(1):121-136

Bruns TE, Tortorelli DA (2001), Topology optimization of nonlinear elastic structures and compliant mechanisms. Comp Meth Appl Mech Eng 190:3443-3459

Cai K (2011), A simple approach to find optimal topology of a continuum with tension-only or compression-only material. Struct Multidiscip Optim 43:827-835

Cheng HC, Kikuchi N (1994), An improved approach for determining the optimal orientation of orthotropic material. Struct Optim 8:101-112

Cheng HC, Pedersen N (1997), On sufficiency conditions for optimal design based on extremnm principles of mechanics. J Mech Phys Solids 45:135-150

Cuomo M, Ventura G (1998), Complementary energy approach to contac problems based on consistent augmented Lagrangian formulation. Math Comput Modelling 28(4-8):185-204

Cuomo M, Ventura G (2000), Complementary energy formulation of no tension masonry-like solids. Comp Meth Appl Mech Eng 189(1):313-339

Diaz AR, Bendsøe MP (1992), Shape optimization of structures for multiple loading conditions using a homogenization method. Struct Optim 4:17-22

Del Piero G (1989), Constitutive equation and compatibility of the external loads for linear elastic masonry-like materials. Meccanica 24(3):150-162

Duysinx P, Bendsøe MP (1998), Topology optimization of continuum structures with local stress constraints. Int J Numer Methods Eng 43: 1453-1478

Eschenauer HA, Olhoff N (2001), Topology optimization of continuum structures: A review. Appl Mech Rev 54:331-389.

Foldager J, Hansen JS, Olhoff N (1998), A general approach forcing convexity of ply angle optimization in composite laminates. Struct Optim 16:201-211

Fuschi P, Giambanco G, Rizzo S (1995), Nonlinear finite element analysis of no-tension masonry structures. Meccanica 30(3):233-249

Genna F (1994), An accurate numerical integration scheme for solving structural problems in the presence of a "no-tension" material, Comput Struct 53:253-273

Guest JK, Prévost JH, Belytschko T (2004), Achieving minimum length scale in topology optimization using nodal design variables and projection functions. Int J Numer Methods Eng $61(2): 238-254$

Guest JK (2009), Imposing maximum length scale in topology optimization. Struct Multidiscip Optim 37(5):463-473

Heyman J (1966), The stone skeleton. Int Journ Solids Structures 2:249-279.

Kang Z, Wang YQ (2011), Structural topology optimization based on non-local Shepard interpolation of density field. Comp Meth Appl Mech Eng 200:3515-3525

Le C, Norato J, Bruns TE, Ha C and Tortorelli DA (2010), Stressbased topology optimization for continua. Struct Multidiscip Optim 41:605-620 
Lucchesi M, Padovani C, Pasquinelli G (1995), On the numerical solution of equilibrium problems for elastic solids with bounded tensile strength. Comp Meth Appl Mech Eng 127:37-56.

Lucchesi M, Padovani C, Pasquinelli G (2000), Thermodynamics of no-tension materials. Int Journ Solids Struct 37(45):65816604

Luo JH, Gea HC (1998), Optimal orientation of orthotropic materials using an energy based method. Struct Optim 15:230-236

Luo Y, Kang Z (2012), Topology optimization of continuum structures with Drucker-Prager yield stress constraints. Comput Struct 90-91:65-75

Luo Z, Zhang N, Wang Y, Gao W (2013), Topology optimization of structures using meshless density variable approximants. Int Journ Solids Struct 93:443-464

Maier G, Nappi A (1990), A theory of discretized structural systems. Eng Struct 12(4):227-234

Pedersen N (1989), On optimal orientation of orthotropic materials. Struct Optim 1:101-106

Pedersen N (1990), Bounds on elastic energy in solids of orthotropic materials. Struct Optim 2:55-63

Querin OM, Victoria M, Marti P (2010), Topology optimization of truss-like continua with different material properties in tension and compression. Struct Multidiscip Optim 42:25-32

Rovati M, Taliercio A (2003), Stationarity of the strain energy density for some classes of anisotropic solids. Int J Solids Struct 40(22):6043-6075

Rozvany GIN, Zhou M, Birker T (1992), Generalized shape optimization without homogenization. Struct Optim 4: 250-254

Romano G, Sacco E (1984), Sul calcolo di strutture non resistenti a trazione. Proc VII AIMETA National Congress, Solids and Structures Mechanics, Trieste, Italy, 217-226.

Sigmund O (2007), Morphology-based black and white filters for topology optimization. Struct Multidiscip Optim 33(45):401-424

Sigmund O, Petersson J (1998), Numerical instabilities in topology optimization: a survey on procedures dealing with checkerboards, mesh-dependencies and local minima. Struct Optim 16:68-75

Suzuki K, Kikuchi N (1991), A homogenization method for shape and topology optimization. Comp Meth Appl Mech Engrg 93:291-318

Svanberg K (1987), Method of moving asymptotes - A new method for structural optimization. Int J Numer Methods Eng 24:359-373

Swan CC, Arora JS (1997), Topology design of material layout in structured composite of high stiffness and high strength. Struct Optim 13:45-59 